PROCEEDINGS OF THE AMERICAN MATHEMATICAL SOCIETY

Volume 124, Number 11, November 1996

\title{
ON THE POSSIBILITIES FOR PARTITIONING A CAKE
}

\author{
JULIUS B. BARBANEL
}

(Communicated by Andreas R. Blass)

\begin{abstract}
We wish to consider the following type of cake division problem: There are $p$ individuals. Each individual has available a measure that he or she uses to evaluate the sizes of pieces of cake. We wish to partition our cake into $q$ pieces in such a way that the various evaluations that the individuals make of the sizes of the pieces satisfy certain pre-assigned equalities and inequalities. Our main result yields a quite general criterion for showing that certain such partitions exist. Following the proof, we consider various applications.
\end{abstract}

\section{The MAIn ReSUlt}

We assume for the remainder of the paper that $m_{1}, m_{2}, \ldots, m_{p}$ are countably additive, nonatomic probability measures, all defined on some common $\sigma$-algebra of subsets of our cake $C$. Whenever a subset of $C$ is mentioned, we shall assume that the subset is a member of this $\sigma$-algebra.

For background material on cake division, the reader is referred to [3] or [4].

Definition. Let Dep (for "Dependency") be the set of all true linear equations involving any of the measures $m_{1}, m_{2}, \ldots, m_{p}$. For example, if $m_{2}(A)=.5 m_{1}(A)+$ $.5 m_{3}(A)$ for all $A \subseteq C$, then " $m_{2}=.5 m_{1}+.5 m_{3}$ " is one of the equations in Dep.

Definition. Suppose that $\mathcal{T}=\left[t_{i j}\right]_{i \leq p ; j \leq q}$ is a $p \times q$ matrix of real numbers. $\mathcal{T}$ is proper if and only if:

1. Each row sums to 0 . That is, for each $i \leq p, t_{i 1}+t_{i 2}+\cdots+t_{i q}=0$.

2. Each column of $\mathcal{T}$ is consistent with the equations in Dep. That is, for each equation in Dep and each $j \leq q$, the given equation holds with $m_{1}, m_{2}, \ldots, m_{p}$ replaced by $t_{1 j}, t_{2 j}, \ldots, t_{p j}$ respectively.

We are now ready to state our main result:

Theorem. Suppose $\mathbf{s}=\left\langle s_{1}, s_{2}, \ldots, s_{q}\right\rangle$ is a sequence of positive real numbers whose sum is 1 , and that $\mathcal{T}=\left[t_{i j}\right]_{i \leq p ; j \leq q}$ is a proper matrix. Then there exists an ordered partition $\mathcal{A}=\left\langle A_{1}, A_{2}, \ldots, A_{q}\right\rangle$ of $C$ such that for some $k>0, m_{i}\left(A_{j}\right)=s_{j}+k t_{i j}$ for each $i \leq p$ and $j \leq q$.

We shall need the following two results:

1. Lyapounov's Theorem (see [4] or [6]): $\left\{\left\langle m_{1}(A), m_{2}(A), \ldots, m_{p}(A)\right\rangle: A \subseteq C\right\}$ is a closed and compact subset of $\mathbb{R}^{p}$.

Received by the editors October 3, 1994 and, in revised form, May 19, 1995.

1991 Mathematics Subject Classification. Primary 28A60; Secondary 90D06.

(C)1996 American Mathematical Society 
2. Dvoretsky, Wald, and Wolfovitz's Theorem (see [5]): $\left\{\left[m_{i}\left(A_{j}\right)\right]_{i \leq p ; j \leq q}\right.$ : $\left\langle A_{1}, A_{2}, \ldots, A_{q}\right\rangle$ is an ordered partition of $\left.C\right\}$ is a closed and convex subset in the space of all $p \times q$ matrices.

It is not hard to see that Lyapounov's result follows from Dvoretsky, Wald, and Wolfovitz's result.

Before beginning the proof, we give some intuition behind the theorem. Suppose $\mathbf{s}=\left\langle s_{1}, s_{2}, \ldots, s_{q}\right\rangle$ is a sequence of real numbers whose sum is 1 . It follows from Lyapounov's Theorem (see, for example, [4]), that there exists an ordered partition $\mathcal{A}=\left\langle A_{1}, A_{2}, \ldots, A_{q}\right\rangle$ of $C$ such that for all $i \leq p$ and $j \leq q, m_{i}\left(A_{j}\right)=s_{j}$. Suppose instead that we wish to deviate from these equalities by the amount given by the proper matrix $\mathcal{T}=\left[t_{i j}\right]_{i \leq p ; j \leq q}$. In other words, we would like to find an ordered partition $\mathcal{A}=\left\langle A_{1}, A_{2}, \ldots, A_{q}\right\rangle$ of $C$ such that for each $i \leq p$ and $j \leq q, m_{i}\left(A_{j}\right)$ differs from $s_{j}$ by exactly $t_{i j}$. This may not be possible. (Note, for example, that we have placed no restrictions on the size of the $t_{i j}$.) However, we can obtain an ordered partition $\mathcal{A}=\left\langle A_{1}, A_{2}, \ldots, A_{q}\right\rangle$ of $C$ such that for all $i \leq p$ and $j \leq q$, all of the $m_{i}\left(A_{j}\right)$ differ from $s_{j}$ by $t_{i j}$ times some fixed positive constant $k$.

Let $D$ be the set mentioned in the result of Dvoretsky, Wald, and Wolfovitz. That is, $D=\left\{\left[m_{k}\left(A_{j}\right)\right]_{i \leq p ; j \leq q}:\left\langle A_{1}, A_{2}, \ldots, A_{q}\right\rangle\right.$ is an ordered partition of $\left.C\right\}$. We shall use the fact that the convex set $D$ is closed under convex combinations. In other words, if $Z_{1}, Z_{2}, \ldots, Z_{q} \in D$ and $\alpha_{1}, \alpha_{2}, \ldots, \alpha_{q}$ are nonnegative real numbers whose sum is 1 , then $\alpha_{1} Z_{1}+\alpha_{2} Z_{2}+\cdots+\alpha_{q} Z_{q} \in D$.

We shall also need the following result, which we established in [1]:

If the measures $m_{1}, m_{2}, \ldots, m_{r}$ are linearly independent and $E=\left\{\left\langle m_{1}(A)\right.\right.$, $\left.\left.m_{2}(A), \ldots, m_{r}(A)\right\rangle: A \subseteq C\right\}$, then for any real number $u$ with $0<u<1$, the point $\langle u, u, \ldots, u\rangle$ is an interior point of $E$ in $\mathbb{R}^{r}$.

Proof of the theorem. Assume that $\mathbf{s}=\left\langle s_{1}, s_{2}, \ldots, s_{q}\right\rangle$ is a sequence of positive real numbers whose sum is 1 , and that $\mathcal{T}=\left[t_{i j}\right]_{i \leq p ; j \leq q}$ is a proper matrix. We must obtain an ordered partition $\mathcal{A}=\left\langle A_{1}, A_{2}, \ldots, A_{q}\right\rangle$ of $C$ such that for some $k>0$, $m_{i}\left(P_{j}\right)=s_{j}+k t_{i j}$ for each $i \leq p$ and $j \leq q$. We shall obtain the desired partition by taking an appropriate convex combination of elements of $D$.

By renumbering if necessary, we may assume that for some $r \leq p$, the set $\left\{m_{1}, m_{2}, \ldots, m_{r}\right\}$ is linearly independent, but each of the measures $m_{r+1}, m_{r+2}, \ldots$, $m_{p}$ can be expressed as a linear combination of the measures in this set. By the previously stated result from [1], if $E=\left\{\left\langle m_{1}(A), m_{2}(A), \ldots, m_{r}(A)\right\rangle: A \subseteq C\right\}$, then for any real number $u$ with $0<u<1$, the point $\langle u, u, \ldots, u\rangle$ is an interior point of $E$ in $\mathbb{R}^{r}$.

For each $j \leq q-1$, we wish to define $B_{j} \subseteq C$. (Our definition will make sense for $j=q$, but this set will not be needed.) The $B_{j}$ 's will be used to define a certain collection of elements of $D$, and we shall obtain our desired element of $D$, and thus our desired partition, by taking a convex combination of these elements.

Fix some real number $s$ such that $1-s_{q}<s<1$. We note that since $1-s_{q}=$ $s_{1}+s_{2}+\cdots+s_{q-1}$, it follows that for each $j \leq q-1, s_{j}<s$.

We know that $\langle s, s, \ldots, s\rangle$ is an interior point of $E$ in $\mathbb{R}^{r}$. Let $\varepsilon>0$ be such that there exists a neighborhood of $\langle s, s, \ldots, s\rangle$ in $\mathbb{R}^{r}$ of radius $\varepsilon$ which lies completely inside of $E$. Fix some real number $k>0$ such that $\max \left\{\left(\frac{k s}{s_{j}}\right)\left|\left\langle t_{1 j}, t_{2 j}, \ldots, t_{r j}\right\rangle\right|: j \leq\right.$ $q-1\}<\varepsilon$. 
For each $j \leq q-1$, our choice of $\varepsilon$ and then of $k$ implies that $\langle s, s, \ldots, s\rangle+$ $\left(\frac{k s}{s_{j}}\right)\left\langle t_{1 j}, t_{2 j}, \ldots, t_{r j}\right\rangle \in E$. Thus, by the definition of $E$, it follows that for each $j \leq q-1$, there exists $B_{j} \subseteq C$ such that $m_{i}\left(B_{j}\right)=s+\left(\frac{k s}{s_{j}}\right) t_{i j}$ for each $i \leq r$.

Claim. For each $i \leq p$ and $j \leq q-1, m_{i}\left(B_{j}\right)=s+\left(\frac{k s}{s_{j}}\right) t_{i j}$.

Proof of Claim. We have just shown that the claim holds for all $i \leq r$ and $j \leq q-1$. If $r=p$, then we are done. Suppose then that $r<p$, and fix some $i$ with $r<i \leq p$. By assumption, there exist constants $\alpha_{1}, \alpha_{2}, \ldots, \alpha_{r}$ such that $m_{i}=\alpha_{1} m_{1}+\alpha_{2} m_{2}+\cdots+\alpha_{r} m_{r}$. Since $m_{1}(C)=m_{2}(C)=\cdots=m_{r}(C)=1$, it follows that $\alpha_{1}+\alpha_{2}+\cdots+\alpha_{r}=1$. Also, since each column of $\mathcal{T}$ is consistent with every equation in Dep, it follows that $t_{i j}=\alpha_{1} t_{1 j}+\alpha_{2} t_{2 j}+\cdots+\alpha_{r} t_{r j}$. Then, for each $j \leq q-1$, we have:

$$
\begin{aligned}
m_{i}\left(B_{j}\right) & =\alpha_{1} m_{1}\left(B_{j}\right)+\alpha_{2} m_{2}\left(B_{j}\right)+\cdots+\alpha_{r} m_{r}\left(B_{j}\right) \\
& =\alpha_{1}\left[s+\left(\frac{k s}{s_{j}}\right) t_{1 j}\right]+\alpha_{2}\left[s+\left(\frac{k s}{s_{j}}\right) t_{2 j}\right]+\cdots+\alpha_{r}\left[s+\left(\frac{k s}{s_{j}}\right) t_{r j}\right] \\
& =\left(\alpha_{1}+\alpha_{2}+\cdots+\alpha_{r}\right) s+\left(\frac{k s}{s_{j}}\right)\left(\alpha_{1} t_{1 j}+\alpha_{2} t_{2 j}+\cdots+\alpha_{r} t_{r j}\right) \\
& =s+\left(\frac{k s}{s_{j}}\right) t_{i j} .
\end{aligned}
$$

This establishes the claim.

We next specify certain elements of $D$. For each $j \leq q$, we define a $p \times q$ matrix $Z_{j}$ as follows:

1. For $j \leq q-1, Z_{j}$ is the matrix with

(a) $j^{t h}$ column equal to $\left[m_{i}\left(B_{j}\right)\right]_{i \leq p}$,

(b) $q^{\text {th }}$ column equal to $\left[m_{i}\left(C \backslash B_{j}\right)\right]_{i \leq p}$,

(c) all other columns consisting of all 0's.

2. $Z_{q}$ is the matrix with

(a) $q^{\text {th }}$ column consisting of all 1's,

(b) all other columns consisting of all 0's.

It is easy to see that $Z_{j}$ is in $D$, since, for each $j \leq q-1, Z_{j}$ arises from the ordered partition $\left\langle\emptyset, \ldots, \emptyset, B_{j}, \emptyset, \ldots, \emptyset, C \backslash B_{j}\right\rangle$, where the " $B_{j}$ " is in the $j$ position, and $Z_{q}$ arises from the ordered partition $\langle\emptyset, \ldots, \emptyset, C\rangle$.

Consider the real numbers $\frac{s_{1}}{s}, \frac{s_{2}}{s}, \ldots, \frac{s_{q-1}}{s}, 1-\left(\frac{s_{1}+s_{2}+\cdots+s_{q-1}}{s}\right)$. These numbers sum to 1 . The first $q-1$ numbers are clearly positive. The last number is also positive since $1-\left(\frac{s_{1}+s_{2}+\cdots+s_{q-1}}{s}\right)=1-\left(\frac{1-s_{q}}{s}\right)=\frac{s-\left(1-s_{q}\right)}{s}$ and, by assumption, $s>1-s_{q}$. We define

$$
Z=\left(\frac{s_{1}}{s}\right) Z_{1}+\left(\frac{s_{2}}{s}\right) Z_{2}+\cdots+\left(\frac{s_{q-1}}{s}\right) Z_{q-1}+\left[1-\left(\frac{s_{1}+s_{2}+\cdots+s_{q-1}}{s}\right)\right] Z_{q} .
$$

Since $Z_{1}, Z_{2}, \ldots, Z_{q} \in D$ and $D$ is convex, it follows that $Z \in D$. Hence, for some ordered partition $\mathcal{A}=\left\langle A_{1}, A_{2}, \ldots, A_{q}\right\rangle$ of $C, Z=\left[m_{i}\left(A_{j}\right)\right]_{i \leq p ; j \leq q}$.

In order to establish the theorem, we wish to show that the two matrices $\left[m_{i}\left(A_{j}\right)\right]_{i \leq p ; j \leq q}$ and $\left[s_{j}+k t_{i j}\right]_{i \leq p ; j \leq q}$ are equal. Since $\left[m_{i}\left(A_{j}\right)\right]_{i \leq p ; j \leq q} \in D$, we know that every row of this matrix sums to 1 . Similarly, since $s_{1}+s_{2}+\cdots+s_{q}=1$ and, for each $i \leq p, t_{i 1}+t_{i 2}+\cdots+t_{i q}=0$, we know that every row of the matrix $\left[s_{j}+k t_{i j}\right]_{i \leq p ; j \leq q}$ sums to 1 . Thus it suffices to show that the first $q-1$ columns 
of these two matrices agree, since it will then follow easily that the last columns of these matrices agree. Fix some $j \leq q-1$. We must show that for each $i \leq p$, $m_{i}\left(A_{j}\right)=s_{j}+k t_{i j}$.

Since the $j^{\text {th }}$ column of $Z_{j}$ is $\left[m_{i}\left(B_{j}\right)\right]_{i \leq p}$ and the $j^{\text {th }}$ column of each the matrices $Z_{1}, Z_{2}, \ldots, Z_{j-1}, Z_{j+1}, \ldots, Z_{q}$ consists of all 0 's, it follows from the definition of $Z$ that the $j^{\text {th }}$ column of $Z$ is $\left(\frac{s_{j}}{s}\right)\left[m_{i}\left(B_{j}\right)\right]_{i \leq p}$. This implies that for each $i \leq$ $p, m_{i}\left(A_{j}\right)=\left(\frac{s_{j}}{s}\right)\left[m_{i}\left(B_{j}\right)\right]$ and hence, by the Claim, $m_{i}\left(A_{j}\right)=s_{j}+k t_{i j}$, as desired.

This completes the proof of the theorem.

\section{Corollaries}

In [1], we defined and studied the notion of super envy-freeness. Super envyfreeness is a strengthening of the notions of fairness and envy-freeness. For background on these and related notions, we refer the reader to [3]. An ordered partition $\left\langle A_{1}, A_{2}, \ldots, A_{p}\right\rangle$ of $C$ is said to be super envy-free with respect to the sequence of measures $\left\langle m_{1}, m_{2}, \ldots, m_{p}\right\rangle$ if for every $i \leq p, m_{i}\left(A_{i}\right)>\frac{1}{p}$, and for every $i, j \leq p$ with $i \neq j, m_{k}\left(A_{j}\right)<\frac{1}{p}$. In [1], we established that there exists such a partition if and only if our measures are linearly independent. We show that one direction (the harder direction) of this result follows from the present theorem.

Corollary 1 (see [1]). If $m_{1}, m_{2}, \ldots, m_{p}$ are linearly independent, then there exists a super envy-free partition of $C$.

Proof. Assume that $m_{1}, m_{2}, \ldots, m_{p}$ are linearly independent. Let $\mathcal{T}=\left[t_{i j}\right]_{i \leq p ; j \leq p}$ be the $p \times p$ matrix with entries along the diagonal equal to 1 and with all other entries equal to $-\frac{1}{p-1}$. Clearly, each row of $\mathcal{T}$ sums to 0 and, since our measures are linearly independent, Dep $=\emptyset$. Hence, $\mathcal{T}$ is a proper matrix. Applying the theorem with $\mathbf{s}=\left\langle\frac{1}{p}, \frac{1}{p}, \ldots, \frac{1}{p}\right\rangle$, we obtain an ordered partition $\mathcal{A}=\left\langle A_{1}, A_{2}, \ldots, A_{p}\right\rangle$ of $C$ such that for some $k>0$ :

$$
m_{i}\left(A_{i}\right)=\frac{1}{p}+k \quad \text { for each } i \leq p
$$

and

$$
m_{i}\left(A_{j}\right)=\frac{1}{p}-\frac{k}{p-1} \quad \text { for each } i, j \leq p \text { with } i \neq j .
$$

Thus, we have $m_{i}\left(A_{i}\right)>\frac{1}{p}$ for each $i \leq p$, and $m_{i}\left(A_{j}\right)<\frac{1}{p}$ for each $i, j \leq p$ with $i \neq j$. Hence, $\mathcal{A}$ is super envy-free with respect to the sequence of measures $\left\langle m_{1}, m_{2}, \ldots, m_{p}\right\rangle$. This establishes Corollary 1 .

We note that the partition obtained above has the property that each person judges his or her own piece of cake to be the same size as everyone else judges their own piece of cake. In other words, $m_{1}\left(A_{1}\right)=m_{2}\left(A_{2}\right)=\cdots=m_{p}\left(A_{p}\right)$. An ordered partition satisfying this property is said to be equitable.

For our second corollary, we show that one of the theorems which we established in [2] follows from the present theorem.

Corollary 2 (see [2]). Assume that the measures $m_{1}, m_{2}, \ldots, m_{p}$ are not identical and that $s_{1}, s_{2}, \ldots, s_{p}$ are positive real numbers with $s_{1}+s_{2}+\cdots+s_{p}=1$. For each $i \leq p$, let $R_{i}$ be one of the relations $<,=$, or $>$. Then there exists an ordered partition $\mathcal{A}=\left\langle A_{1}, A_{2}, \ldots, A_{p}\right\rangle$ of $C$ such that for each $i \leq p, m_{i}\left(A_{i}\right) R_{i} s_{i}$. 
Proof. By renumbering if necessary, we assume that $m_{p-1} \neq m_{p}$. Fix $s_{1}, s_{2}, \ldots, s_{p}$ as in the statement of the corollary. We wish to define an appropriate proper matrix $\mathcal{T}=\left[t_{i j}\right]_{i \leq p ; j \leq p}$ and to then apply the theorem.

For each $i \leq p$, define $t_{i i}$ as follows:

$$
\begin{array}{ll}
t_{i i}=-1 & \text { if } R_{i} \text { is the }<\text { relation, } \\
t_{i i}=0 & \text { if } R_{i} \text { is the }=\text { relation, } \\
t_{i i}=1 & \text { if } R_{i} \text { is the }>\text { relation. }
\end{array}
$$

We note that we need only show that we can define the rest of $\mathcal{T}$ in such a way that $\mathcal{T}$ is a proper matrix. It will then follow easily from the theorem that the desired partition exists.

For each $i \leq p$ and $j \leq p-2$ with $i \neq j$, define $t_{i j}$ arbitrarily, subject to the constraint that each of the $p-2$ columns that have so far been defined is consistent with each of the equations in Dep.

Next, we note that there is only one as yet undefined entry in each of the last two rows $\left(t_{p-1 p}\right.$ in row $p-1$, and $t_{p p-1}$ in row $\left.p\right)$. Define these two values in the unique way such that each row sums to 0 . We note that " $m_{p-1}=m_{p}$ " is not one of the equations in Dep, since we have assumed that $m_{p-1} \neq m_{p}$. Hence, the assignment of values to $t_{p-1 p-1}, t_{p p-1}, t_{p-1 p}$ and $t_{p p}$ that we have made is not inconsistent with any equation in Dep.

It remains for us to define $t_{i j}$ for $i \leq p-2$ and $j=p-1, p$. For each $i \leq p-2$, define $t_{i p-1}$ arbitrarily, subject to the constraint that the $p-1^{\text {st }}$ column of $\mathcal{T}$ is consistent with each of the equations in Dep.

We have only to complete defining the $p^{t h}$ column of $\mathcal{T}$. For each $i \leq p-2$ we simply define $t_{i p}$ to be the unique number such that the $i^{t h}$ row sums to 0 .

It is immediate from our construction that each row of $\mathcal{T}$ sums to 0 . It is also immediate that each of the first $p-1$ columns is consistent with each equation in Dep. These two facts together imply that the $p^{\text {th }}$ column is consistent with each equation in Dep. Hence, $\mathcal{T}$ is a proper matrix.

Finally, it is clear from our definition of the $t_{i i}$ for $i \leq p$ that if $\mathcal{A}=\left\langle A_{1}, A_{2}, \ldots\right.$, $\left.A_{p}\right\rangle$ is the ordered partition which we obtain by applying the theorem to the matrix $\mathcal{T}$ and the sequence $\left\langle s_{1}, s_{2}, \ldots, s_{p}\right\rangle$, then for each $i \leq p, m_{i}\left(P_{i}\right) R_{i} s_{i}$, as desired. This establishes Corollary 2.

Our third corollary establishes that, if our measures are linearly independent, we can obtain an ordered partition such that each individual believes that the relative sizes of pieces of the partition satisfy any previously set ordering, where the orderings for different individuals can be chosen independently.

Corollary 3. Assume that $m_{1}, m_{2}, \ldots, m_{p}$ are linearly independent. For each $i \leq$ $p$, let $\mathbf{z}^{i}=\left\langle z_{i 1}, z_{i 2}, \ldots, z_{i q}\right\rangle$ be some ordering of the integers $\{1,2, \ldots, q\}$. Then there exists an ordered partition $\mathcal{A}=\left\langle A_{1}, A_{2}, \ldots, A_{q}\right\rangle$ of $C$ such that for each $i \leq p, m_{i}\left(A_{z_{i 1}}\right)<m_{i}\left(A_{z_{i 2}}\right)<\cdots<m_{i}\left(A_{z_{i q}}\right)$.

Proof. Let $\mathcal{T}=\left[t_{i j}\right]_{i \leq p ; j \leq q}$ be the matrix of real numbers such that each row sums to 0 and, for each $i \leq p, t_{i z_{i 1}}<t_{i z_{i 2}}<\cdots<t_{i z_{i q}}$. Since $m_{1}, m_{2}, \ldots, m_{p}$ are linearly independent, Dep $=\emptyset$. It follows that $\mathcal{T}$ is a proper matrix. Let $\mathcal{A}=$ $\left\langle A_{1}, A_{2}, \ldots, A_{q}\right\rangle$ be the ordered partition of $C$ obtained by applying the theorem to $\mathcal{T}$ and the sequence $\left\langle\frac{1}{p}, \frac{1}{p}, \ldots, \frac{1}{p}\right\rangle$. We claim that this $\mathcal{A}$ satisfies the corollary.

Fix some $i \leq p$ and $j<j^{\prime} \leq q$. We must show that $m_{1}\left(A_{z_{i j}}\right)<m_{i}\left(A_{z_{i j^{\prime}}}\right)$. Let $k$ be as in the statement of the theorem. Then $k>0, m_{i}\left(A_{z_{i j}}\right)=\frac{1}{p}+k t_{i z_{i j}}$, 
and $m_{i}\left(A_{z_{i j^{\prime}}}\right)=\frac{1}{p}+k t_{i z_{i j^{\prime}}}$. Also, since $j<j^{\prime}$, we know that $t_{i z_{i j}}<t_{i z_{i j^{\prime}}}$. Hence $m_{i}\left(A_{z_{i j}}\right)<m_{i}\left(A_{z_{i j^{\prime}}}\right)$, as desired. This establishes Corollary 3.

If there were linear dependency relationships between our measures, then certain orderings of the type considered in Corollary 3 would be impossible. As a simple example, suppose that $m_{2}=.5 m_{1}+.5 m_{3}$. Then it would certainly not be possible to obtain an ordered partition $\left\langle A_{1}, A_{2}, \ldots, A_{q}\right\rangle$ of $C$ with $m_{1}\left(A_{1}\right)<$ $m_{1}\left(A_{2}\right), m_{2}\left(A_{1}\right)>m_{2}\left(A_{2}\right)$, and $m_{3}\left(A_{1}\right)<m_{3}\left(A_{2}\right)$.

We also point out that, in contrast to the situation involving rows given in Corollary 3, we do not have the freedom to order columns arbitrarily, even if we assume that our measures are linearly independent. In other words, it is not always possible to find an ordered partition of $C$ such that for each fixed piece of the partition, the $p$ individuals' relative judgments of the size of that piece satisfy a particular ordering. For example, it is certainly not possible to find an ordered partition $\left\langle A_{1}, A_{2}, \ldots, A_{q}\right\rangle$ of $C$ such that for each $j \leq q, m_{1}\left(A_{j}\right)<m_{2}\left(A_{j}\right)$, since this would imply that $m_{1}(C)<m_{2}(C)$.

We close this section by noting that additional structure can be built into the kinds of relationships that we have been discussing. For example, let us suppose that in Corollary 3, one of the conditions that we wish to have our ordered partition $\left\langle A_{1}, A_{2}, \ldots, A_{q}\right\rangle$ satisfy is $m_{1}\left(A_{7}\right)<m_{1}\left(A_{8}\right)<m_{1}\left(A_{9}\right)$. By carefully choosing the entries in our matrix $\mathcal{T}$, we could have obtained such a partition satisfying that $m_{1}\left(A_{7}\right)<m_{1}\left(A_{8}\right)<m_{1}\left(A_{9}\right)$ and also that $m_{1}\left(A_{9}\right)-m_{1}\left(A_{8}\right)=$ $17\left[m_{1}\left(A_{8}\right)-m_{1}\left(A_{7}\right)\right]$.

\section{Relation matrices AND AN Open QUeStion}

In this section we discuss a general type of problem concerning the existence of ordered partitions satisfying certain relationships. We shall consider in what sense our theorem solves problems of this type.

Definition. A pq relation matrix is a $p \times q$ matrix, each of whose entries is one of the relations $<,=$, or $>$. If $\mathcal{R}=\left[R_{i j}\right]_{i \leq p ; j \leq q}$ is a $p q$ relation matrix, $\mathbf{s}=\left\langle s_{1}, s_{2}, \ldots, s_{q}\right\rangle$ is a sequence of positive real numbers whose sum is 1 , and $\mathcal{A}=\left\langle A_{1}, A_{2}, \ldots, A_{q}\right\rangle$ is an ordered partition of $C$, we shall say that $\mathcal{A}$ satisfies $\mathcal{R}$ with respect to $\mathbf{s}$, if and only if for each $i \leq p$ and $j \leq q, m_{i}\left(A_{j}\right) R_{i j} s_{j}$. Also, a proper matrix $\mathcal{T}=\left[t_{i j}\right]_{i \leq p ; j \leq q}$ satisfies $\mathcal{R}$ if and only if for each $i \leq p$ and $j \leq q, t_{i j} R_{i j} 0$.

Suppose $\mathcal{R}$ is a $p q$ relation matrix and $\mathbf{s}=\left\langle s_{1}, s_{2}, \ldots, s_{q}\right\rangle$ is a sequence of positive real numbers whose sum is 1 . It follows in a straightforward manner from our theorem that if there exists a proper matrix satisfying $\mathcal{R}$, then there exists an ordered partition $\mathcal{A}$ which satisfies $\mathcal{R}$ with respect to $\mathbf{s}$. Clearly the converse of this statement is true, since if $\mathcal{A}=\left\langle A_{1}, A_{2}, \ldots, A_{q}\right\rangle$ is an ordered partition which satisfies $\mathbf{s}$ with respect to $\mathcal{R}$, then $\mathcal{T}=\left[m_{i}\left(A_{j}\right)-s_{j}\right]_{i \leq p ; j \leq q}$ is a proper matrix which satisfies $\mathcal{R}$. Hence, the question of whether there exists an ordered partition which satisfies $\mathcal{R}$ with respect to $\mathbf{s}$ is equivalent to the question of whether there exists a proper matrix which satisfies $\mathcal{R}$.

We next give an example to illustrate the use of this idea to establish the existence of some desired partition. Suppose that we wish to show that there exists an ordered 
partition $\mathcal{A}=\left\langle A_{1}, A_{2}, A_{3}, A_{4}\right\rangle$ of $C$ satisfying the following relationships:

$$
\begin{array}{llll}
m_{1}\left(A_{1}\right)>.1 & m_{1}\left(A_{2}\right)>.2 & m_{1}\left(A_{3}\right)<.3 & m_{1}\left(A_{4}\right)=.4 \\
m_{2}\left(A_{1}\right)<.1 & m_{2}\left(A_{2}\right)=.2 & m_{2}\left(A_{3}\right)<.3 & m_{2}\left(A_{4}\right)>.4 \\
m_{3}\left(A_{1}\right)=.1 & m_{3}\left(A_{2}\right)>.2 & m_{3}\left(A_{3}\right)<.3 & m_{3}\left(A_{4}\right)>.4 \\
m_{4}\left(A_{1}\right)>.1 & m_{4}\left(A_{2}\right)>.2 & m_{4}\left(A_{3}\right)<.3 & m_{4}\left(A_{4}\right)>.4
\end{array}
$$

In addition, let us assume that the following linear dependency relationships hold between the measures $m_{1}, m_{2}, m_{3}$, and $m_{4}$ :

$$
m_{3}=.5 m_{1}+.5 m_{2}, \quad m_{4}=.75 m_{1}+.25 m_{2} .
$$

(We assume that this list is exhaustive, except for the linear dependency relationships which are implied by these two.)

Restating the problem using the terminology of this section, we must show that there is an ordered partition $\mathcal{A}=\left\langle A_{1}, A_{2}, A_{3}, A_{4}\right\rangle$ of $C$ which satisfies the relation matrix

$$
\mathcal{R}=\left[\begin{array}{llll}
> & > & < & = \\
< & = & < & > \\
= & > & < & > \\
> & > & < & >
\end{array}\right]
$$

with respect to the sequence $\mathbf{s}=\langle .1, .2, .3, .4\rangle$.

Consider the matrix

$$
\mathcal{T}=\left[\begin{array}{cccc}
.04 & .04 & -.08 & 0 \\
-.04 & 0 & -.04 & .08 \\
0 & .02 & -.06 & .04 \\
.02 & .03 & -.07 & .02
\end{array}\right]
$$

It is straightforward to verify that $\mathcal{T}$ is a proper matrix that satisfies $\mathcal{R}$. Thus, it follows from our discussion above that there is an ordered partition $\mathcal{A}$ of $C$ which satisfies $\mathcal{R}$ with respect to $\mathbf{s}$, as desired.

We note that both Corollary 1 and Corollary 2, but not Corollary 3, fit into this framework. For Corollary $1, \mathcal{R}$ is the $p p$ relation matrix whose entries along the diagonal are all $>$ 's and whose other entries are all $<$ 's, and $\mathbf{s}=\left\langle\frac{1}{p}, \frac{1}{p}, \ldots, \frac{1}{p}\right\rangle$. For any particular example of Corollary 2 , the diagonal entries $\mathcal{R}$ must be specified and the other entries of $\mathcal{R}$ can be chosen as desired, and $s$ must be specified.

Next, we give an example that looks very similar to the example above, but turns out to be quite different. Suppose we wish to show that there exists an ordered partition $\mathcal{A}=\left\langle A_{1}, A_{2}, A_{3}, A_{4}\right\rangle$ of $C$ such that:

$$
\begin{array}{llll}
m_{1}\left(A_{1}\right)>.25 & m_{1}\left(A_{2}\right)=.25 & m_{1}\left(A_{3}\right)<.25 & m_{1}\left(A_{4}\right)>.25 \\
m_{2}\left(A_{1}\right)=.25 & m_{2}\left(A_{2}\right)<.25 & m_{2}\left(A_{3}\right)<.25 & m_{2}\left(A_{4}\right)>.25 \\
m_{3}\left(A_{1}\right)>.25 & m_{3}\left(A_{2}\right)<.25 & m_{3}\left(A_{3}\right)<.25 & m_{3}\left(A_{4}\right)<.25 \\
m_{4}\left(A_{1}\right)>.25 & m_{4}\left(A_{2}\right)<.25 & m_{4}\left(A_{3}\right)>.25 & m_{4}\left(A_{4}\right)>.25
\end{array}
$$

In addition, let us assume that the following linear dependency relationship holds between the measures $m_{1}, m_{2}, m_{3}$, and $m_{4}$ :

$$
m_{1}+m_{2}=m_{3}+m_{4}
$$


We wish to show that there is an ordered partition $\mathcal{A}=\left\langle A_{1}, A_{2}, A_{3}, A_{4}\right\rangle$ of $C$ which satisfies the relation matrix

$$
\mathcal{R}=\left[\begin{array}{llll}
> & = & < & > \\
= & < & < & > \\
> & < & < & < \\
> & < & > & >
\end{array}\right]
$$

with respect to the sequence $\mathbf{s}=\langle .25, .25, .25, .25\rangle$. As in the previous example, it suffices to show that there exists a proper matrix $\mathcal{T}$ which satisfies $\mathcal{R}$.

We observe that there is nothing obvious preventing us from obtaining such a matrix. In particular, the relations in each row are consistent with the necessity of rows summing to 0 (in contrast with, for example, a relation matrix with some row consisting of >'s), and the relations in each column are consistent with the necessity of satisfying the given linear dependency relationship (in contrast with, for example, a relation matrix with some column consisting of $[>,>,<,<])$. However, there is a more subtle difficulty here, which arises from the interaction of these two types of constraints.

A careful examination of $\mathcal{R}$, taking into account the given linear dependency relationship, reveals that if there were a proper matrix $\mathcal{T}$ satisfying $\mathcal{R}$, it would follow that each entry in the first row of this matrix would be strictly larger than the corresponding entry in the third row of this matrix. This contradicts the fact that each row of a proper matrix sums to 0 . Thus, no such proper matrix exists, and hence there does not exist an ordered partition $\mathcal{A}=\left\langle A_{1}, A_{2}, A_{3}, A_{4}\right\rangle$ of $C$ such that the given 16 relations are satisfied.

These last two examples lead us to the question of how one could determine whether or not there exists a proper matrix which satisfies a given relation matrix, or, equivalently (by the theorem), how one could determine whether or not there exists an ordered partition which satisfies a given relation matrix with respect to a given sequence. In general, how does one obtain a proper matrix as in the first of our two examples, and how does one show that no such proper matrix exists, as in our second example? We do not know the answer, and so we close with the following:

Open problem. Suppose $\mathbf{s}=\left\langle s_{1}, s_{2}, \ldots, s_{q}\right\rangle$ is a sequence of positive real numbers whose sum is 1 and $\mathcal{R}=\left[R_{i j}\right]_{i \leq p ; j \leq q}$ is a relation matrix. Find a procedure for determining whether there exists an ordered partition which satisfies $\mathcal{R}$ with respect to $\mathbf{s}$. Or, equivalently (by the theorem), find a procedure for determining whether there exists a proper matrix which satisfies $\mathcal{R}$.

\section{ACKNOWLEDGMENT}

We wish to thank Alan Taylor, William Zwicker, and the referee for many helpful suggestions on an earlier version of this paper.

\section{REFERENCES}

1. J. B. Barbanel, Super envy-free cake division and independence of measures, to appear in the Journal of Mathematical Analysis and Applications 197 (1996), pp. 54-60.

2. J. B. Barbanel and W. Zwicker, Two applications of a theorem of Dvoretsky, Wald, and Wolfovitz to cake division, pre-print.

3. S. J. Brams and A. D. Taylor, An envy-free cake division protocol, American Mathematical Monthly 102 (1995), pp. 9-18. 
4. L. E. Dubins and E. H. Spanier, How to cut a cake fairly, American Mathematical Monthly 68 (1961), pp. 1-17. MR 23:B2068

5. A. Dvoretsky, A. Wald, and J. Wolfovitz, Relations among certain ranges of vector measures, Pacific Journal of Mathematics 1 (1951), pp. 59-74. MR 13:331f

6. A. Lyapounov, Sur les fonctions-vecteurs completement additives, Bull Acad. Sci. URSS 6 (1940), pp. 465-478.

Department of Mathematics, Union College, Schenectady, New York 12308

E-mail address: barbanej@gar.union.edu 